\title{
Genetic and Environmental Effects on Tonic Immobility Duration of Red-Winged Tinamou Applying Survival Analysis
}

\section{-Author(s)}

\section{Hata ME' \\ Caetano SL' \\ Boleli ICIII \\ Queiroz SA}

Departamento de Zootecnia, Faculdade de Ciências Agrárias e Veterinária, Universidade Estadual Paulista "Júlio Mesquita Filho".

" Departamento de Estatística, Faculdade de Ciências Agrárias e Veterinária, Universidade Estadual Paulista "Júlio Mesquita Filho".

III Departamento de Morfologia e Fisiologia Animal, Faculdade de Ciências Agráriase Veterinárias, Universidade Estadual Paulista "Júlio Mesquita Filho".

\section{-Mail Address}

Corresponding author e-mail address Milene Elissa Hata

Departamento de Zootecnia - Faculdade de Ciências Agrárias e Veterinárias Campus de Jaboticabal, UNESP. Via de acesso Prof Paulo Castelane $s / n^{\circ}$, Jaboticabal, 14884900, São Paulo, Brazil.

Tel: (16) 981639321

Email: milenehata@gmail.com

\section{- Keywords}

Animal welfare; Behavioral trait; Genetic parameters; Survival kit; Wild birds.

\section{ABSTRACT}

Fear behavioral studies provide knowledge on animal welfare, and fearful behaviors can be used as selection criteria of individuals adapted to intensive rearing system. The survival analysis methodology was applied to estimate tonic immobility (TI) duration, as an indicator of fear, of red-winged tinamous (Rhynchotus rufescens) reared in captivity and to determine if $\mathrm{Tl}$ is genetically influenced. A number of 539 birds born between 2006 and 2010 were evaluated. The exploratory data analysis was performed using the Kaplan-Meier estimate (KM), and the covariates were then fit to a Cox model, considering month of observation nested within year of birth and body weight as fixed effects and the random effect of sire as frailty term. In order to predict genetic values and to estimate heritability, the model of proportional hazards was applied, using a Weibull distribution as the baseline hazard. Birds born in the last year presented shorter TI duration than those born in the previous year, as shown by the survival KM curves, indicating a decline in fearfulness from one year to the next. The Cox analysis detected that hazard function was reduced as body weight increased. The frailty term was significant $(p<0.05)$, showing that sires induced variation in the $\mathrm{TI}$ duration of the offspring. Heritability estimated as 0.37 , indicating the influence of additive genes. These findings suggest that the selection of for short TI duration may allow reducing fearfulness of a red-winged tinamou population after some generations.

\section{INTRODUCTION}

Red-winged tinamous (Rhynchotus rufescens) are native SouthAmerican birds widely distributed in Brazil (Sick, 1997). Despite being wild, their domestication has attracted great interest due to their high cynegetic value, and excellent carcass yield, particularly breast yield (Queiroz et al., 2013), as well as remarkable adaptation to commercial feeds (Hoshiba et al., 2003). Therefore, aiming at supplying a consumer market for exotic meats, this species could be reared under similar commercial conditions as broilers. However, this requires investigating the behavior of the red-winged tinamou in order to find individuals that are calmer and more adaptable to captivity because, studies about evolution of domestic animals indicate that reduced fearfulness is critical to the adaptation of species to the stressful rearing conditions imposed by humans (Campler et al., 2009).

Fear is still the main cause of injuries in animal production systems, suggesting that domestic animals are still not fully habituated to captivity (Al-Aqil et al., 2013). The degree of fear may be evaluated the duration of tonic immobility (TI) presented by an animal, and it is a natural behavior expressed by an animal under threat, when nervous stimuli transitionally block its motor system (Miyatake et al., 2009). 
The longer the TI duration, the greater is the animal's fearfulness (Mills \& Faure, 2000). Genetic parameters estimated for $\mathrm{TI}$ using linear models have shown that this trait present sufficient additive genetic variation. Therefore, survival analysis methodology may aid the analysis of $\mathrm{TI}$.

Selection based on fearful behavioral traits may enhance animal productivity by reducing the expression of undesirable fear responses. Pelhaitrea et al. (2012) verified that the selection of poultry for high productivity has resulted in correlated negative welfare responses, particularly during the process of domestication of chickens, when the selection of layers for higher egg production caused social and emotional behavioral changes. Therefore, the inclusion of behavioral traits in genetic improvement programs may be an interesting strategy, since it has been shown that genetics may be used to improve animal welfare (Agnvall et al., 2012).

The study of some behavioral traits, including $\mathrm{TI}$, may aid the understanding of the domestication process of wild species, such as the red-winged tinamou (Rhynchotus rufescens), as well as the selection of less fearful animals, leading to the establishment of populations less reactive to humans and better adapted to captivity. Tonic immobility duration is commonly measured in individual birds, which are induced to a catatonic state by the researcher. However, birds are allowed to remain in IT only for a determined period, after which they are removed from this condition if they do not naturally recover. When an individual reaches the maximum possible value, i.e., it exceeds maximum TI duration, the datum is referred censored and it is best evaluated using the survival analysis (Colosimo \& Giolo, 2006). The objective of this study was to identify sources of variation that affect $\mathrm{Tl}$ duration in red-winged tinamous reared in captivity, to apply the method of survival analysis to fit the model to be used in genetic analyses, and to estimate the heritability of this trait.

\section{MATERIAL AND METHODS}

\section{Bird and data management}

The descriptive data used in this study were obtained in red-winged tinamous (Rhynchotus rufescens) reared in captivity in the Wild Bird Sector of the Department of Animal Science of UNESP-FCAV, state of São Paulo, Brazil, where TI was also measured. The birds were distributed in $20 \mathrm{~m}^{2}$ pens, at a density of approximately 15 birds per pen, in a house similar to those used commercial poultry production, with concrete floor covered with Cynodon dactylon grass litter. Drinking water and feed were supplied ad libitum. The housed was daily cleaned by handlers, which otherwise had limited access and contact with the birds to avoid stress.

TI measurements were performed in a quite location distant from the rearing area in order to reduce the influence of environmental factors on data collection. Birds were removed from the rearing shed, and taken to a room located beside the breeding shed. Before the observations, birds were weighed and individually maintained in polyvinyl chloride (PVC) crates for 10 minutes. The method of Jones \& Faure (1981) was applied to measure TI. Each bird was held on its back on a V-shaped wood structure to induce TI. The duration of TI was measured using a chronometer $($ Technos ${ }$, model YP2151/8P) by an observer standing at a 1-m distance from the bird. Maximum TI duration was established as 1200 seconds (s). Therefore, TI duration of the birds that did not recover up to 1,200 s were considered as censored measurements.

\section{Covariates}

Bird sex, year of birth, month, age, and weight at the time of $\mathrm{TI}$ measurement were recorded and used as covariates to explain differences in $\mathrm{Tl}$ duration. Individuals with no siblings and/or unknown sires were not included in the analyses, as this information is needed for genetic analysis. A total of 539 redwinged tinamous were included in the database. The effect of year of birth (YB) indicated the breeding cycle during which the bird was born. The breeding season of $R$. rufescens starts in September and continues until April of the following year (Bruneli et al., 2005). Tonic immobility results of birds born between 2006 and 2010 were recorded, corresponding to four years of birth: YB1 = from September 2006 till April 2007; YB2 = from September 2007 till April 2008; YB3 = from September 2008 till April 2009; and YB4 = from September 2009 till April 2010. Tonic immobility duration was measured in different months, except for the period between August and October, and this effect was established as the covariate "month" (M).

At the time of $\mathrm{TI}$ measurement, bird age ranged between 41 and 263 days. Bird age was divided into classes for non-parametric analysis purposes. The logrank test was applied in the survival analysis to rank the birds according to age. Firstly, the influence of the onset of breeding activity (around 240 days of age) on TI duration was evaluated by comparing the survival 
curve of the group of birds with age (mean \pm standard deviation) equal or higher than 240 days (252.00 \pm 6.10) with that of birds with up to 239 days of age $(125.06 \pm 38.60)$. The results were not significant at $5 \%$ probability level. Only 16 birds, corresponding to $2.78 \%$ of the total population, were older than 240 days; however, according to Colosimo \& Giolo (2006) class imbalance does not affect survival analysis results. The best ranking of birds according to age class (mean \pm standard deviation) was: Group $1=$ birds between 41 and 139 days of age (99.45 \pm 15.82$)$ and Group 2 $=$ birds between 140 and 263 days of age (175.96 \pm 31.12). The same procedure was applied to establish body weight classes (mean \pm standard deviation) as Group 1 = body weight between 196 and 638 grams (g) $(482.81 \pm 101.48)$, and Group $2=640$ to $882 \mathrm{~g}$ (718.16 \pm 51.32).

\section{Kaplan-Meier estimate}

Data files were prepared and submitted to exploratory analysis using SAS statistical software (Statistical Analysis System, version 9.2). The procedure LIFETEST was used for the survival analysis, generating non-parametric estimates, as well as the survival function $(\hat{s}(t))$ curves of the response parameter $\mathrm{TI}$ duration determined by the Kaplan-Meier (KM) estimate, described as:

$$
\hat{s}(t)=\prod_{j=t_{j}<t}\left(1-\frac{d_{j}}{n_{j}}\right)
$$

where: $t_{1}<t_{2} \ldots<t_{k}$ corresponds to $k$ different and increasing TI duration times; $d_{j}$ corresponds to the number of birds recovering from $\mathrm{Tl}$ in $t_{j}, j=1, \ldots, k$; $n_{j}$ corresponds to the number of birds at risk of $t_{j}$, i.e., those that did not recover from $\mathrm{TI}$ and were not censored until the time immediately before $t_{j}$.

The failure time corresponded to the period during which the birds remained in $\mathrm{TI}$, and therefore, survival curves $\hat{s}(t)$ were built for TI duration of the groups established for each covariate. In order to test the hypothesis that curves were not different, the logrank test was applied, assuming a Chi-square distribution $\left(\chi^{2}\right)$ with one degree of freedom and $5 \%$ significance level when comparing two groups. The hazard ratio $(h(t))$ was also obtained by the LIFETEST procedure of SAS statistical package, and determined the number of red-winged tinamous likely to recover from $\mathrm{TI}$ every $60 \mathrm{~s}$. This measure is useful to determine the likelihood of birds recovering from $\mathrm{Tl}$ over time, i.e., the number of birds remaining in Tl every $60 \mathrm{~s}$ allowed determining the number of birds recovering from $\mathrm{Tl}$ in the previous minute until the end of the observation period (1200s).

\section{Cox model and frailty term}

Routines of the R statistical software (version 2.13.0 Copyright(C) 2011; The R Foundation for Statistical Computing) were applied for the semi-parametric analysis, using Cox regression to model the hazard function. This methodology uses regression parameters similar to those applied in generalized linear models. The general expression of Cox regression is:

$$
\lambda(t)=\lambda_{0}(t) \exp \left\{\chi^{\prime} \beta\right\}
$$

where: $\lambda_{0}(t)$ represents the baseline hazard; $\exp \left\{\chi^{\prime} \beta\right\}=\exp \left\{\chi_{1} \beta_{1}+\ldots+\chi_{p} \beta_{p}\right\} ; \quad \chi$ represents the vector of covariate values, and $\beta$, the vector of parameters associated to the covariates.

The Cox regression method was applied to test the models based on the previous analyses performed using the KM estimate. In order to select the model that best explained the variations in TI duration, the partial likelihood ratio test (LRT) was applied. Aiming at testing proportional hazard ratio and complying with the basic assumption for the use of regression in the Cox model, Schoenfeld standardized residues were calculated. Finally, the gamma frailty model was also tested by the Cox model in the multivariate context. The frailty term corresponded to the random effect of breeder, i.e., of the sires of the birds submitted to TI measurement. Frailty was introduced in the Cox model as follows:

$$
\lambda_{i j}(t)=Z_{i} \lambda_{0}(t) \exp \left\{x_{i j}^{\prime} \beta\right\}
$$

where: $\lambda_{i i}(t)$ is the hazard function; $X_{i i}^{\prime}$ is the $p$-dimensional vector of the covariates; $\lambda_{0}(t)$ is an unknown baseline hazard function; $\beta$ is the $p$-dimensional vector of unknown coefficients of regression; and $\mathrm{Z}_{i}$ are the frailty values that follow a distribution with mean $=1$ and variance $\sigma^{2}$.

\section{Genetic analysis}

The genetic analysis of the TI trait was performed using the software Survival Kit V6.0 (Ducrocq et al., 2010), developed to assist animal breeding programs. Fixed and random covariates, which were previously analyzed and defined using the KM estimator and fit to the Cox model, were included. In addition, bird, sex, dam and sire records in the pedigree file were included to design the relationship matrix. The following Cox proportional hazard model was applied:

$$
\lambda_{i j}(t)=\lambda_{0}(t) \exp \left\{x_{i j}^{\prime} \beta+z(t)^{\prime} u\right\}
$$


where the vectors $\lambda_{i j}(t)$ describe the hazard function of a bird to recover from $\mathrm{Tl}$, i.e., the probability of recovering at time $t$, considering it was immobile immediately before this time; $\lambda_{0}(t)$ is the baseline hazard; $X_{i j}^{\prime}$ are the fixed covariates; $\beta$ is an unknown vector of coefficients of regression; $z(t)^{\prime}$ is the random coefficient of sire with the corresponding vector of $u$ parameters.

The random covariate $z(t)^{\prime}$ described a multivariate normal distribution, in which the covariance structure among individuals was modeled using the genetic relationship matrix to obtain the genetic additive values of the individuals. It was assumed that $\lambda_{0}(t)$ presented a Weibull distribution, because the study of the curves indicated that this was the most adequate distribution to describe the TI behavior, according to the dispersion of $\mathrm{TI}$ duration. Therefore, $\lambda_{0}(t)=\lambda \rho(\lambda t)^{p-1}=\rho t^{p-1} \exp (\rho 1 n \lambda)$, where the shape and scale parameters $\lambda$ and $\rho$ present Weibull distribution, respectively, at a defined time t. The genetic values were essential to calculate the variance among sires $\left(\sigma_{s}^{2}\right)$ and to estimate TI heritability, according to the equation of Yazdi et al., (2006):

$$
h^{2}=\frac{4 \sigma_{s}^{2}}{\sigma_{s}^{2}+1}
$$

Finally, the predicted reliability of the Weibull model $\left(R_{\text {weibull }}\right)$ was calculated using non-censored measurements $\left(n_{\text {ncens }}\right)$ and $\sigma_{s}^{2}$. Reliability determines the accuracy of the breeding values predicted by the Weibull model. The adopted convergence criterion was $10^{-8}$ and the pedigree file contained data of 637 birds.

\section{RESULTS}

\section{Kaplan-Meier estimate}

The survival curves obtained by KM estimate show different TI failure times over the four tested years of birth. Survival was higher in the YB1 population than in the YB3, indicating that TI duration increased from the first to the third year of birth, and it was reduced in the last year of birth (YB4), which in turn was similar to that observed in YB2 (Figure 1). The hazard ratio $(h(t))$ indicates that the number of birds exposed to hazard over time is equivalent to the number of individuals that remained in $\mathrm{Tl}$ after some individuals recovered in the previous 60 seconds. Therefore, the lower the number of birds exposed to hazard per minute, the higher the number of birds recovering from $\mathrm{TI}$, and consequently, the less fearful is the analyzed population. In Figure 1 , considering ratios of birds that recovered from $\mathrm{TI}$ $50 \%$ or higher relative to the total population, the YB1 red-winged tinamous were the least fearful, as they recovered the fastest (180 s), followed by YB2 and YB4 (240 s), whereas YB3 birds took the longest to the recover (300 s). However, the comparison of survival curves using the logrank test for every pair of years of birth showed that only YB1 birds were different from the others at $5 \%$ probability level, indicating that the sole inclusion of this covariate in the model is not sufficient to explain the changes in time.

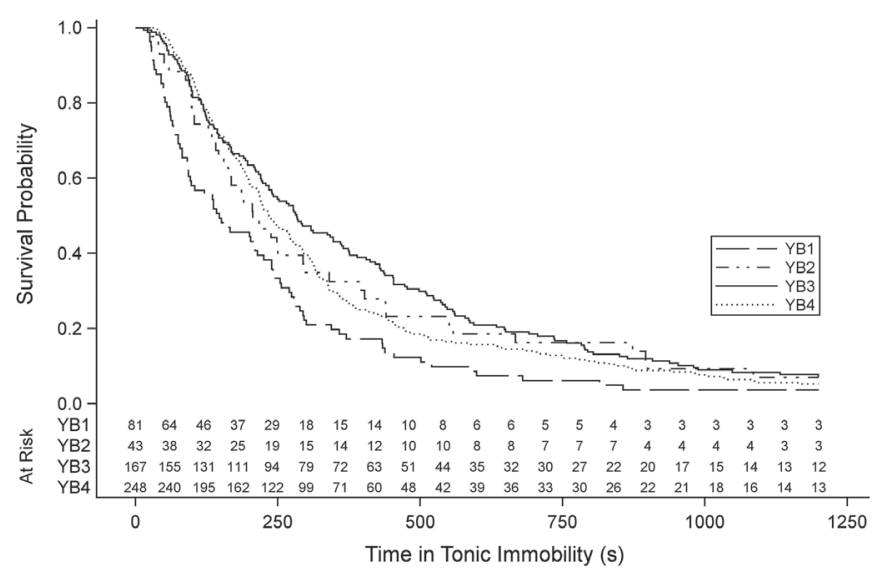

Figure 1 - Survival function curves of tonic immobility duration estimated by Kaplan- Meier with number of red-winged tinamous (Rhynchotus rufescens) at risk as a function of year of birth (YB). YB1 = birds generated during the breeding cycle of September 2006 to April 2007; YB2 = September 2007 to April 2008; YB3 = September 2008 to April 2009; YB4 = September 2009 to April 2010

The logrank test showed significant differences $(p<0.05)$ among $\mathrm{TI}$ survival curves when comparing the same months (M) of $\mathrm{TI}$ measurement among different years of birth (YB). Therefore, we decided to nest the effect of month within year of birth, establishing a new covariate (MYB) to study their joint effect on TI duration. Thelogrank test also determined different $\hat{s}(t)$ for covariate ages at $5 \%$ significance level $(p=0.02)$, indicating the eldest group were more fearful than the youngest group. In addition, the hazard ratio decreased faster over time in the younger birds, with at least half of the group recovering from $\mathrm{TI}$ before 240 $\mathrm{s}$. The same proportion of birds recovering from $\mathrm{TI}$ was reached at $300 \mathrm{~s}$ in eldest group (Figure 2 ). The $\hat{s}(t)$ obtained by the KM estimate were similar for females and males $(p=0.4358)$, showing no differences in $\mathrm{TI}$ duration between sexes. Lastly, the analysis of the covariate body weight showed that the heavier birds remained longer in $\mathrm{Tl}$, and the hazard ratio values determined that $50 \%$ of the heavier birds recovered in $300 \mathrm{~s}$, whereas more than $50 \%$ of the lighter birds recovered in $240 \mathrm{~s}$ (Figure 3). 


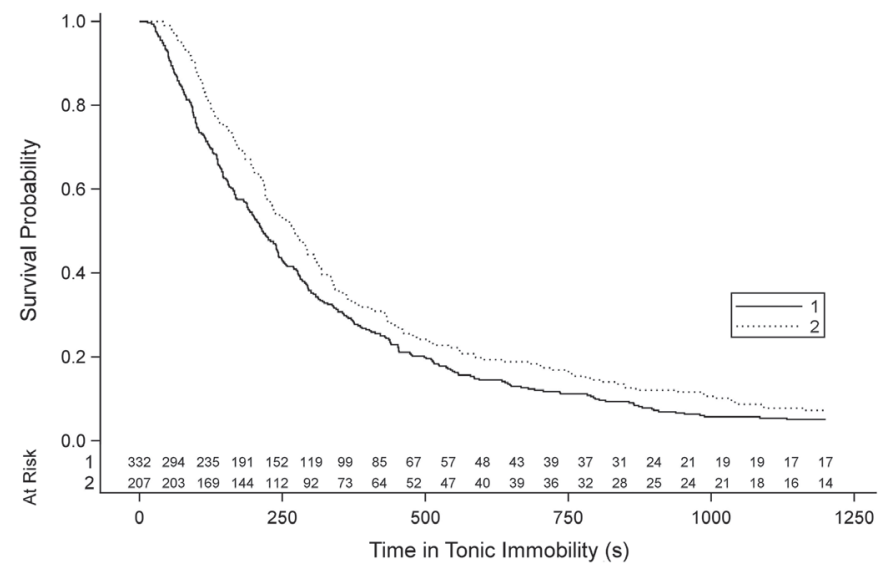

Figure 2 - Survival function curves of tonic immobility duration estimated by Kaplan-Meier with number of red-winged tinamous (Rhynchotus rufescens) at risk as a function of age. Group $1=40-139$ days of age. Group $2=140-263$ days of age

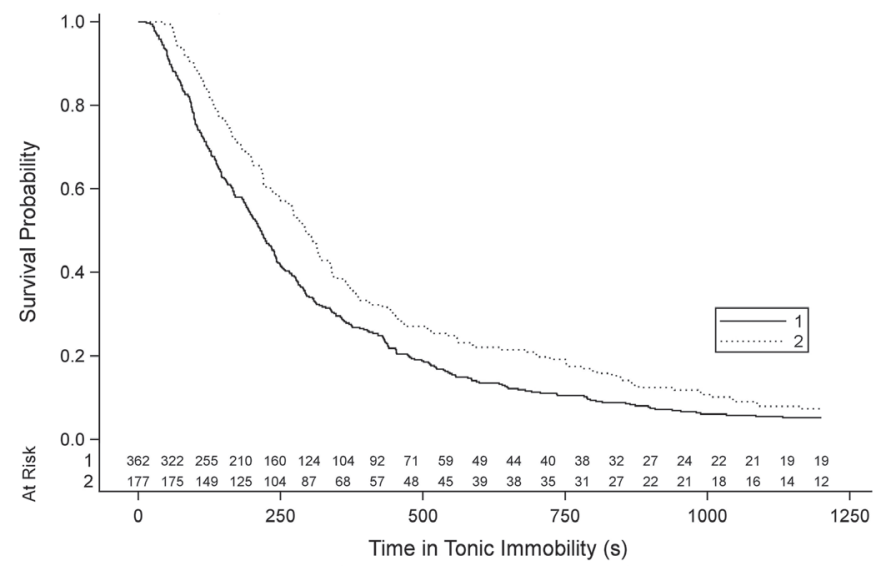

Figure 3 - Survival function curves of tonic immobility duration estimated by Kaplan-Meier with number of red-winged tinamous (Rhynchotus rufescens) at risk as a function of body weight. Group $1=196-639 \mathrm{~g}$. Group $2=640-882 \mathrm{~g}$

\section{Cox model and frailty term}

The covariates previously analyzed by the KM estimate were tested using Cox regression. Therefore, different models were built using the covariates month nested in year of birth (MYB), body weight, and age at the time of $\mathrm{TI}$ measurement. However, due to the strong correlation between bird age and body weight $(r=0.7507)$, only one of these covariates should be used, and this decision was made using the LRT. The obtained LRT values of $0.16(p=0.6915)$ when body weight was excluded from the model, and of 6.72 $(p=0.0095)$ when age was excluded, determined the inclusion of the covariate body weight in the model. The inclusion of both covariates (body weight and age) in the model was tested, but the result showed a nonsignificant effect $(p<0.05)$ of age.

Therefore, the Cox model including the covariates MYB and body weight (BW) presented the best fit to explain variations in $\mathrm{TI}$ duration. The explanatory power $\left(R^{2}\right)$ of the model was $10.6 \%$, and the estimated probability of agreement was $60.3 \%$. The values of all coefficients were negative, indicating that both MYB and body weight reduced failure time, i.e., birds took longer to recover from TI. This indicates that the heavier red-winged tinamous remained longer in $\mathrm{TI}$, and therefore, were more fearful. Hence, the survival and hazard functions estimated for the fitted model were determined as, respectively:

$$
\begin{aligned}
& \hat{s}(t \mid x)=\left[\hat{S}_{0}(t)\right]^{\exp \left\{\hat{\beta}_{M Y B}+\left(\hat{\beta}_{B W} \times B W\right)\right\}} \\
& \hat{\lambda}(t \mid x)=\hat{\lambda}_{0}(t) \exp \left\{\hat{\beta}_{M B Y}+\left(\hat{\beta}_{B W} \times B W\right)\right\}
\end{aligned}
$$

As expected, the inclusion of frailty in the Cox model did not significantly change the effects or the interpretation of the fixed effects present in the model. However, it showed a significant association between TI duration and sire $\left(p=0.013\right.$ with $\chi^{2}=39.47$ and $\sigma^{2}=0.082$ ), indicating differences among sires. In addition, the agreement probability of the model was $65.9 \%$, with an explanatory power $\left(R^{2}\right)$ of $20.4 \%$. Frailty estimates $\left(Z_{i}\right)$ serve as baseline hazards weights, according to the equation $\lambda_{i j}(t)=Z_{i} \lambda_{0}(t) \exp \left\{x_{i j}^{\prime} \beta\right\}$, indicating that $Z_{i}$ values equal or very close to 1 do not significantly affect the hazard, whereas values higher than 1 indicate that the hazard increases (Figure 4). The $Z_{i}$ values obtained for 86 different sires ranged between 0.66 and 1.38 , demonstrating the possibility of reducing fear in red-winged tinamous by selecting birds that recover faster from $\mathrm{Tl}$.

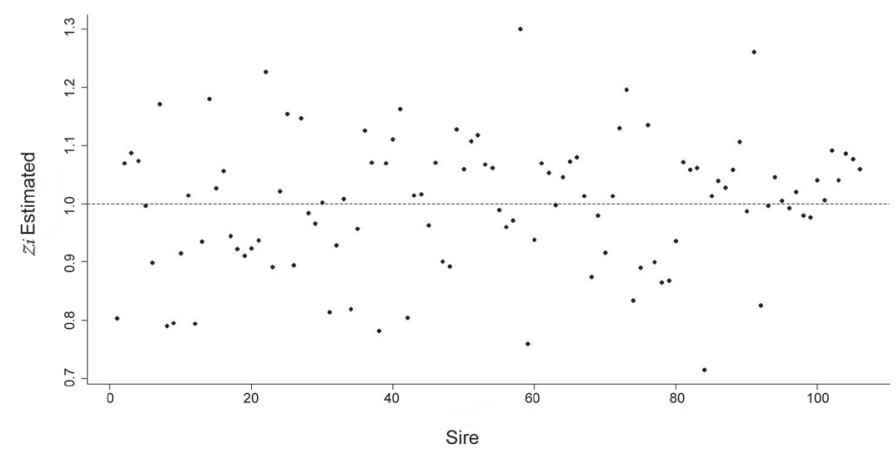

Figure 4 - Estimates of frailty parameters $\left(Z_{i}\right)$ obtained by including sire information in the Cox model

\section{Genetic analysis}

The inclusion of the effect of sire in the model used for the analysis of $\mathrm{TI}$ duration showed this trait is influenced by additive genes, which allowed performing the genetic analysis using the software Survival Kit V6.0 (Ducrocq et al., 2010). The Survival Kit allowed identifying the group represented by the effect of the month of May (5) nested in the last year 
of birth (YB4) as the baseline group, i.e., the group with the highest number of birds that recovered from TI within this MYB class (Table 1). The YB1 birds evaluated in January presented higher probability of recovering from $\mathrm{Tl}$ relative to the baseline group, with a hazard ratio of 4.1. The YB2 birds evaluated in April presented a 0.09 hazard ratio, i.e., their probability of recovering from $\mathrm{TI}$ was approximately 11.11-fold $(1 / 0.09)$ lower relative to the baseline group. Body weight was estimated as -0.0019 , with a standard error of \pm 0.00071 , indicating that heavier birds have lower probability of recovering from $\mathrm{Tl}$, and that for each $1 \mathrm{~g}$ of increase in body weight, the risk of failure is reduced in $0.19 \%$.

Table 1 - Estimates of the model of proportional hazards, adopting the Weibull distribution for the baseline hazard for tonic immobility duration of red-winged tinamous (Rhynchotus rufescens), as determined by the survival analysis.

\begin{tabular}{lcccc}
\hline Covariate & Estimate & Standard error & Hazard ratio & Failure \\
\hline YB1 Month 1 & 1.4135 & 0.5529 & 4.110 & 6 \\
\hline YB1 Month 2 & 0.2129 & 0.5307 & 1.237 & 8 \\
\hline YB1 Month 3 & -1.0376 & 0.8048 & 0.354 & 4 \\
YB1 Month 4 & -0.3435 & 0.5536 & 0.709 & 6 \\
\hline YB1 Month 5 & -0.0123 & 0.4052 & 0.988 & 17 \\
\hline YB1 Month 7 & 0.5383 & 0.3845 & 1.713 & 24 \\
\hline YB1 Month 10 & -0.8594 & 0.5347 & 1.351 & 7 \\
\hline YB1 Month 12 & 0.8214 & 0.6035 & 0.423 & 6 \\
\hline YB2 Month 2 & -0.3735 & 0.4008 & 0.688 & 16 \\
YB2 Month 3 & -0.4976 & 0.3851 & 0.608 & 14 \\
\hline YB2 Month 4 & -2.3943 & 0.7219 & 0.091 & 3 \\
YB2 Month 6 & 0.1892 & 0.5676 & 1.208 & 7 \\
\hline YB3 Month 1 & -0.3488 & 0.2459 & 0.706 & 39 \\
\hline YB3 Month 2 & -0.8516 & 0.2489 & 0.427 & 50 \\
\hline YB3 Month 3 & -1.1878 & 0.6388 & 0.305 & 4 \\
\hline YB3 Month 4 & -1.0388 & 0.3513 & 0.354 & 18 \\
\hline YB3 Month 11 & -0.1229 & 0.2461 & 0.884 & 23 \\
\hline YB3 Month 12 & -0.3159 & 0.2998 & 0.729 & 21 \\
\hline YB4 Month 4 & -0.3248 & 0.2094 & 0.723 & 35 \\
\hline YB4 Month 5 & 0.000 & - & 1.000 & 135 \\
\hline YB4 Month 7 & 0.3011 & 0.1702 & 1.351 & 65 \\
\hline Weight & -0.0019 & 0.0007 & - & 508 \\
\hline YB = yer bith & & & & \\
\hline
\end{tabular}

$Y B=$ year of birth; $Y B 1=$ September 2006 to April 2007; YB2 = September 2007 to April 2008; YB3 = September 2008 to April 2009; YB4 = September 2009 to April 2010

As shown in Table 2, the value of the Weibull shape parameter $(\rho)$ was 1.32 , indicating that the risk of recovery increased as TI duration increased. The intercept, also defined as the general mean of failure time ( $\rho 1 n \lambda)$, was equal to -6.44 . These results describe the baseline risk function and the survival function $\hat{s}(t)=\exp \left\{-(\lambda t)^{\rho}\right\}$. Genetic variance among breeders was equal to 0.103 , and the estimated heritability was 0.37, indicating moderate heritability. The predicted
Table 2 - Parameters estimated for tonic immobility duration of red-winged tinamous (Rhynchotus rufescens) according the survival analysis.

\begin{tabular}{lc}
\hline Parameters & TI \\
\hline Heritability & 0.37 \\
Intercept $(\rho \mid n \lambda)$ and standard error & $-6.44(0.32)$ \\
Shape $(\rho)$ of Weibull distribution and standard error & $1.32(0.04)$ \\
Scale $(\lambda)$ of Weibull distribution & 0.0076 \\
Predicted reliability of the Weibull model $\left(R_{\text {weibull }}\right)$ & 0.98 \\
\hline
\end{tabular}

reliability (accuracy) of 0.93 obtained in the genetic evaluation, as determined by the Weibull model $\left(R_{\text {weibull }}\right)$, was also very significant. Lastly, Figure 5 shows that there was a wide dispersion among expected progeny differences among birds, indicating the possibility of selecting the sires of future generations.

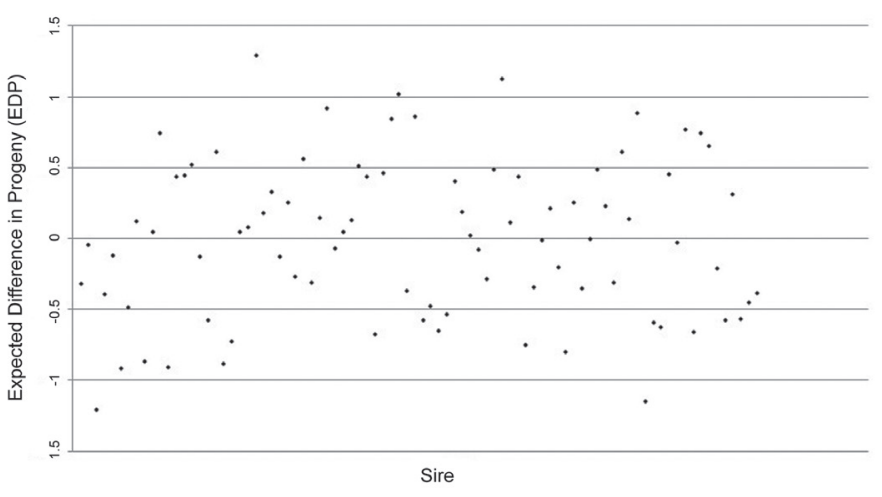

Figure 5 - Expected progeny tonic immobility differences in red-winged tinamous (Rhynchotus rufescens), according to sire

\section{DISCUSSION}

The calculated Kaplan-Meier estimates showed shorter Tl duration in red-winged tinamous born in the last year evaluated (YB4) compared with those born in the third year (YB3), indicating that the evaluated population may habituate to human presence and to intensive rearing environments. This result also shows that the species $R$. rufescens can be domesticated, as animals better adapted to captivity tend to be less fearful and to present higher fitness potential, i.e., higher potential of mating and producing a progeny relative to their wild counterparts (Campler et al., 2009).

The survival curves and the logrank test results showed that bird sex does not have any significant effect (0.05) on Tl duration in red-winged tinamous reared in captivity. In fact, because males brood the eggs and care for the progeny during the first weeks of life, it was expected that their TI duration would be lower than that of females. Opposite results are reported in literature. For instance, TI duration was 
shorter in males than in females in 8-week-old broilers (Akpa et al., 2007). Considering that the breeding system of wild red-winged tinamous is polyandric (Sick, 1997), i.e., a female mates with several different males and the latter brood and care for the progeny, the influence of sex on fear behavior in this species may be different from that of domestic poultry due to behavioral and hierarchical differences.

Relative to age, the survival curve showed that older birds remained longer in TI. Studies have demonstrated that poultry are capable of forming memories (Jones et al., 2002) and that, under threat, fear may activate the birds' memory, increasing their state of alertness (Belzung \& Filippot, 2007). Therefore, older red-winged tinamous were more fearful perhaps because they had experienced situations in the captivity environment as aversive, such as daily management practices, or because they get more anxious as they were not able to express their natural behavior. Another possible reason why young red-winged tinamous are more sociable and less fearful than adults is that they need to share the common environment with their conspecifics and are under the protection of the father. However, as they age, they become more fearful because they need to face threats individually.

Finally, the non-parametric analysis indicated that heavier red-winged tinamous are more fearful than the lighter ones. This finding is consistent with those reported by Ghareeb \& Böhm (2008), who also observed that heavy broilers were more fearful when submitted to the TI test at 5 and 6 weeks of age. On the other hand, a study with Japanese quails showed that birds selected for high body weight presented shorter TI duration compared with those selected for low body weight (Jones et al., 1997). Anderson \& Jones (2012), evaluating the effect of genetic selection on the growth performance and fearfulness behavior of Leghorn layers, found that, although selection influenced growth parameters, fearfulness was not affected. However, as older birds are also heavier, the effects of these two factors may be confounding. In the population evaluated in the present study, the estimated Pearson's correlation between age and body weight was $0.7507(p=<0.0001)$, i.e., these two covariates presented high positive correlation. This indicates that the longer TI duration of the heavy redwinged tinamous may be due to the fact that they were older. Therefore, the LRT was used to choose which of these covariates should be included in the Cox model, which results showed a gradual increase in TI duration as body weight increased.
The primary objective of evaluating TI duration in $R$. rufescens reared in captivity in the present study was to select the least fearful individuals to aid the domestication of this species, as less fearful animals are capable of adapting to the presence of humans and to captivity conditions, with consequent welfare and productivity benefits (Belyaev, 1979). Therefore, the fact that the heavier red-winged tinamous presented longer $\mathrm{Tl}$ duration may indicate that selecting less fearful individuals to be reared in captivity may have negative effects on productivity as a result of the selection of lighter birds, thereby reducing the average body weight of the population. However, Price (2002) observed that morphological changes are one of the consequences of the process of domestication, and reported that some animals, such as domestic ducks (Anas platyrhynchos domesticus), present smaller body size relative to their wild counterparts. This may be explained by their lighter skeleton and smaller brain size as a result of physiological changes because, in captivity, animals have easy access to food and are protected from predators.

Therefore, in species that are not fully domesticated, body weight reduction after a few generations could be considered as a natural process, and the reduced fearfulness would indicate adaptation to the captivity environment. This suggests that sometime during the domestication process, lighter animals would remain in TI for a shorter period than the heavier ones. However, considering the studied population, the mere fact that the lighter birds presented shorter TI duration does not allow inferring that they are being domesticated because other factors, such as reproduction, affect this process.

The fit of the survival and risk functions, as estimated by the Cox model, was efficient to differentiate TI duration among red-winged tinamous reared in captivity. It should be noted that the birds born in YB4 were the least fearful, as determined by the greatest hazard of recovering from TI. This demonstrates that this population of red-winged tinamous has possibly begun to adapt to the captivity environment, habituating to the human presence and to the management system, and therefore, can be domesticated.The frailty term (effect of sire) included in the Cox model was significant $(p<0.05)$, confirming reports on the genetic determination of fearful behavior, suggesting that there is sufficient genetic variation to allow the selection for these behavioral traits. The frailty estimates $\left(\mathbf{Z}_{i}\right)$ allowed identifying less fearful sires (with higher frailty values) that may be 
included in selection programs to generate less fearful progenies. On the other hand, the wide range of $\mathrm{Z}_{i}$ estimates obtained in the present study suggested that not only conventional selection can be applied to select less fearful red-winged tinamous to be reared in captivity, but also divergent selection of sires with lower $\mathrm{Z}_{i}$ values, or more fearful, in genetic programs aimed at repopulating preservation parks and other natural areas.

The genetic analysis performed using survival analysis showed that the estimated heritability value of TI was moderate, evidencing an additive genetic effect, which indicates the possibility of genetic selection for this trait. On the other hand, Mills \& Faure (1991) obtained heritability values between 0.09 and 0.23 in Japanese quails selected for $\mathrm{Tl}$ duration for eight generations. Altan et al. (2005) reported TI heritability estimates of $0.07 \pm 0.07$ for sire lines and $0.55 \pm 0.23$ for dam lines of laying chickens selected for egg production for 12 generations, and suggested that fearfulness can be reduced by selection. In a recent study, Agnavall et al. (2012) selected three generations of the Red Junglefowl (ancestors of domestic chickens) based solely on the reaction in a standardized fear-of-human test, which included $\mathrm{TI}$, and reported a TI heritability estimate of $0.08 \pm 0.08$, which is much lower than that obtained in the present study with red-winged tinamous. These differences may be attributed to the different methodologies applied to calculate heritability, as well as to differences in fearfulness among species. It should be emphasized that understanding the behavior of red-winged tinamous and providing them rearing conditions that take into account their welfare will allow these birds to adapt faster to captivity and favors domestication. As consumers of animal products are increasingly concerned with the welfare of livestock, such practices could increase the interest of consumers in alternative poultry meat.

\section{CONCLUSIONS}

Sex does not affect $\mathrm{Tl}$ duration in red-winged tinamous and therefore, does not need to be considered when analyzing this trait. On the other hand, year of birth, month of TI measurement, and body weight influence TI duration and should be included in the analysis. In addition, additive genes influence $\mathrm{TI}$ duration, as shown by the evaluation of sire effect and by heritability estimates, suggests that it is possible to select less fearful red-winged tinamous reared in captivity to allow their future commercial production. On the other hand, the more fearful red-winged tinamous can be submitted to divergent selection for studies on factors that affect fear in this species.

\section{ACKNOWLEDGEMENTS}

The authors are thankful to the employees of Wild Bird Sector (Department of Animal Sciences, UNESP/FCAV, São Paulo, Brazil) for their technical assistance; to Conselho Nacional de Desenvolvimento Científico e Tecnológico (CNPq) and Coordenação de Aperfeiçoamento de Pessoal de Nível Superior (Capes) for the financial support.

\section{REFERENCES}

Agnvall B, Jöngrn M, Strandberg $E$, Jensen P. Heritability and genetic correlations of fear-related behaviour in Red Junglefowl - possible implications for early domestication. PLoSONE 2012;7(4):e35162.

Akpa GN, Koffi KA, Hassan MR, Kabir M, Duru S, Yashim SM. Effects of feed type, sex and plumage condition on tonic immobility and blood parameters in broilers. International Journal of Poultry Science 2007;6(3):218-222.

Al-Aqil A, Zulkiflii, Bejo MH, Sazili AQ, Rajion MA, Somchit MN. Changes in heat shock protein 70, blood parameters, and fear-related behavior in broiler chickens as affected by pleasant and unpleasant human contact. Poultry Science 2013;92(1):33-40.

Altan Ö, Settar P, Ünver Y, Çabuk M. Heritabilities of tonic immobility and leucocytic response in sire and dam layer lines. Turkey Journal Veterinary Animal Science2005;29(1):3-8.

Anderson KE, Jones DR. Effect of genetic selection on growth parameters and tonic immobility in leghorn pullets. Poultry Science 2012;91(3):765770 .

Belyaev DK. Destabilizing selection as a factor in domestication. The Journal of Heredity 1979;70(5):301-308.

Belzung C, Philippot P. Anxiety from a phylogenetic perspective: is there a qualitative difference between human and animal anxiety? Neural Plasticity 2007. Available from: htpp://doi.org/ urn:doi:10.1155/2007/59676

Bruneli FAT, Tholon P, Isaac FL, Damasceno PR, Tonhati H, Queiroz AS. Caracterização da reprodução de perdizes (Rhynchotus rufescens) em cativeiro. Ars Veterinária 2005;21(2):272-280.

Campler $M$, Jöngren $M$, Jensen $P$. Fearfulness in red junglefowl and domesticated white leghorn chickens. Behavioural Processes 2009;81(1):39-43

Colosimo EA, Giolo SR. Análise de sobrevivência aplicada. In: ABE: Projeto fisher. São Paulo: Edgard Blücher; 2006. p.367.

Der G, Everitt BS. A handbook of statistical analyses using SAS. $2^{\text {nd }}$ ed. Boca Raton: Chapman and Hall; 2001.

Ducrocq V, Sölkner J, Mészáros G. Survival Kit v6 - a software package for survival analysis. Proceedings of the 9th World Congress on Genetics Applied to Livestock Production; 2010; Leipzig, Sachsen. Germany.

Ghareeb K, Böhm J. Fear behaviour, ease of capture and performance traits of growing meat type chickens. International Journal of Poultry Science 2008;7(12):1185-1189. 
Hoshiba MA, Tanaka ALR, Rodrigues GA, Figueiredo G, Tholon P, Junqueira $\mathrm{OM}$, et al. Resultados preliminares do consumo e desperdício de ração com diferentes tamanhos de péletes de perdizes (Rhynchotus rufescens) em cativeiro. Anais da $40^{\mathrm{a}}$ Reunião Anual da Sociedade Brasileira de Zootecnia; 2003; Santa Maria, Rio Grande do Sul. Brasil.

Jones RB, Facchin L, McCorquodale C. Social dispersal by domestic chicks in a novel environment: reassuring properties of a familiar odourant. Animal Behaviour 2002;63(4):659-666.

Jones RB, Satterlee DG, Marks HL. Fear-related behaviour in Japanese quail divergently selected for body weight. Applied Animal Behaviour Science 1997;52(1-2):87-98.

Jones RB. Fear and adaptability in poultry: Insights, implications and imperatives. World Poultry Science Journal 1996;52(2):131-174.

Mills AD, Faure JM. Divergent selection for duration of tonic immobility and social reinstatement behavior in japanese quail (Coturnix coturnix japonica) chicks. Journal of Comparative Psychology 1991;105(1):2538.

Mills $A D$, Faure JM. Ease of capture in lines of japanese quail (Coturnix japonica) subjected to contrasting selection for fear or sociability. Applied Animal Behaviour Science 2000;69(2):125-134
Miyatake T, Nakayama S, Nishi Y, Nakajima S. Tonically immobilised selfish prey can survive by sacrificing others. Proceedings of the Royal Society of London B: Biological Sciences 2009;276(1668):2763-2767.

Pelhaitrea A, Mignon-Grasteaua S, Bertin A. Selection for wheat digestibility affects emotionality and feeding behaviours in broiler chicks. Applied Animal Behaviour Science 2012;139(1):114-122.

Price EO. Animal domestication and behavior. New York: CABI Publishing; 2002.

Queiroz de FA, Carvalho de MM, Sugui JK, Nunes J, Felipe L, Santos dos EC, et al. Meat and carcass traits of the red-winged tinamou (Rhynchotus rufescens). Brazilian Journal of Poultry Science 2013;15(2):113-118.

Sick H. Ordem tinamiformes. In: Ornitologia brasileira. Rio de Janeiro: Nova Fronteira; 1997. p. 153-157.

Yazdi MH, Visscher PM, Ducrocq V, Thompson R. Heritability, reliability of genetic evaluations and response to selection in proportional hazard models. Journal of Dairy Science 2002;85(6):1563-157. 
